\title{
A Proposed Estimation of the Expanded Uncertainty of Charpy Impact Testers
}

\author{
A. Abu-Sinna ${ }^{1}$, Saher R. Hassan ${ }^{2}$ \\ ${ }^{1}$ Force and Material Metrology Laboratory (FMMD), National Institute of Standards (NIS) \\ Giza, Egypt \\ Email: a_abu_sinna [AT] yahoo.com \\ ${ }^{2}$ Force and Material Metrology Laboratory (FMMD), National Institute of Standards (NIS) \\ Giza, Egypt \\ Email: saher_eng [AT] hotmail.com
}

\begin{abstract}
Uncertainty estimation is one of the very delicate tasks in the field of measurements. For the purpose of calibration of Charpy impact testing machines, it is necessary to evaluate and identify the expanded uncertainty. Factors affecting the uncertainty estimations are; the uncertainty of reference force and length measuring devices and its long-term instability (drift), machine resolution, rated energy error, indicated energy error, losses due to the drag of the pointer, friction losses in the bearing and air resistance, and other geometric parameters. In this study, the uncertainty estimation of the Charpy impact machines is based on the direct verification used in the BS DIN ISO 1482 standard.
\end{abstract}

Keywords— Calibration, Impact Testers, Uncertainty, Direct Method.

\section{INTRODUCTION}

Charpy impact testing is a low-cost and reliable test method which is commonly required by the construction codes for fracture-critical structures such as bridges and pressure vessels. Yet, it took from about 1900 to 1960 for impact-test technology and procedures to reach levels of accuracy and reproducibility such that the procedures could be broadly applied as standard test methods.

Without uniformity of test results from day to day and from laboratory to laboratory, the impact test has little meaning. Over the years, researchers have learned that the results obtained from an impact test can depend strongly upon the specimen size and the geometry of the notch, anvils, and striker: To a lesser degree, impact test results also depend upon other variables such as impact velocity, energy lost to the test machine, and friction. The goal of those who have written and modified ASTM Standard Test companies performing acceptance tests are typically required to verify the performance of their impact machine using certified verification specimens [1].

Since 1998, National Institute of Standards, NIS, has entered the facility of the direct verification of Charpy impact testers according to BS DIN ISO 10045 [2] standard. However, and starting from 2017, NIS has changed the reference standard to BS DIN ISO 148-2 [3]. This standard describes two methods:

1- The direct method allowing the physical and geometrical properties of the different parts of the testing machine to be verified statically and separately.

2- The indirect method: global verification method of the pendulum impact testing machine using Charpy $\mathrm{V}$ reference test pieces.

The direct method shall be used, initially, when the machine is being installed or repaired, and if the indirect method gives a doubtable result.

This study concerns describing the uncertainty evaluation method of the direct verification of the Charpy impact testers as applied in NIS. Consequently, this study proposes all sources of error that might affect the uncertainty estimation such as reference load, angle and length measuring devices, resolution effect [4], indicated energy error, drag to of the pointer, and bearing friction. 


\section{UNCERTAINTY ESTIMATION}

The main sources of uncertainty of the direct calibration of Charpy impact testing machines (pendulum impact) are:

\subsection{Uncertainty of reference load, angle and length measuring devices used, $U_{\text {ref }}$}

The potential energy AP of the pendulum Charpy impact tester shall be verified according to the following procedure and shall not differ from the nominal value (AN) by more than $\pm 1.0 \%$ according to [2].

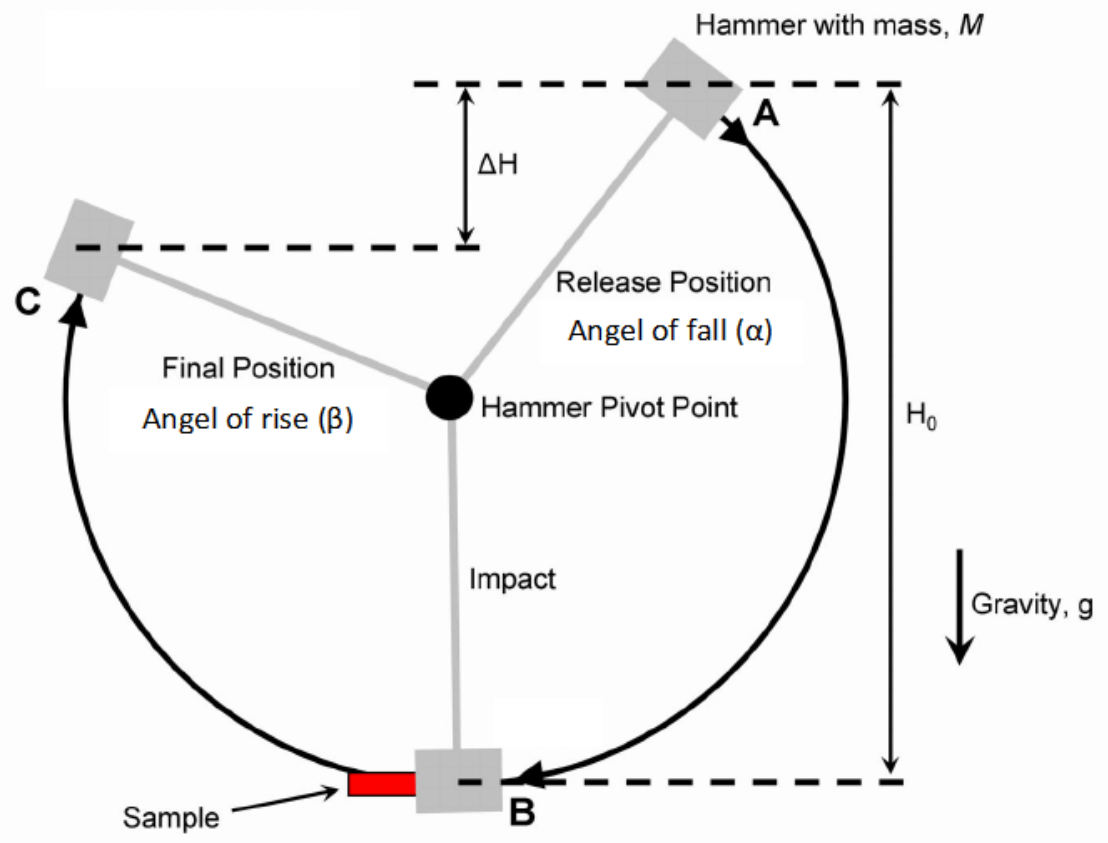

Figure 1: The angles used for the calculation of impact energy [5].

By the nature of its design, the center of gravity of the pendulum is always very close to the striking edge of the pendulum and the straight line which prolongs this edge passes very close to the axis of rotation.

Instead of determining the weight $F_{1}$ of the pendulum and the distance from its center of gravity to the axis, it is easier to determine a force $F$ such that - acting at a known distance $L_{2}$ from the axis - it has the same moment relative to this axis as the weight of the pendulum and $L_{2}$ may be equal to $L$.

To determine the pendulum impact energy, Lift the pendulum so that its center of gravity is in the horizontal plan of the axis of rotation to within a tendency of 15/1000 (i.e. practically that the striking edge is in the horizontal plan of the axis) and support one point of this striker a distance $L_{2}$ from the axis on another horizontal striker perpendicular to the first one and supported by a balance beam or better, by a dynamometer. Measure the force $\mathrm{F}$ exerted by the pendulum on the load measuring device and the distance $L_{2}$ from the anvil to the axis to the nearest $0.2 \%$ as shown in Fig.1. The moment $M$ of the pendulum is equal to:

$$
M=F \times L_{2}
$$

Then measure the angle of rotation described by the pendulum to pass from the rest position to the fall position. This measurement shall be made using a cathetometer or an angle to an accuracy $\pm 0.065^{\circ}$ (see Fig. 1 ). The angle may be greater than $90^{\circ}$. The pendulum energy $E$ is equal to:

$$
E=A_{P}=M(1-\cos \alpha)=F \cdot L_{2}(1-\cos \alpha)
$$

To get the uncertainty of the measuring devices used in the energy measurements:

$$
\begin{aligned}
& \Delta A_{P}=\frac{\partial A_{P}}{\partial F} \Delta F+\frac{\partial A_{P}}{\partial L_{2}} \Delta L_{2}+\frac{\partial A_{P}}{\partial \alpha} \Delta \alpha \\
& \frac{\Delta A_{P}}{A_{P}}=\frac{\Delta F}{F}+\frac{\Delta L_{2}}{L_{2}}+\frac{\Delta \alpha}{\alpha} \cdot \frac{\alpha \cdot \sin \alpha}{(1-\cos \alpha)}
\end{aligned}
$$

Hence, the magnitude of the uncertainties in the three components, $u_{r e f}$, is equal to:

$$
U_{\text {ref }}=\sqrt{U_{F}^{2}+U_{L}^{2}+\left(U_{\alpha} \cdot \frac{\alpha \cdot \sin \alpha}{(1-\cos \alpha)}\right)}
$$




\subsubsection{Uncertainty due to reference load measuring device, $U_{F}$}

The following equation describes the uncertainty of the reference load measuring device:

$$
U_{F}=\sqrt{U_{F s t d}^{2}+U_{d r i f t}^{2}+U_{\text {temp }}^{2}}
$$

The maximum relative expanded uncertainties of the reference force transducers ( $\left.U_{F s t d}\right)$ used for load verifications of the Charpy impact testing machines are given in Table 1, for all of the four force transducers classes specified in EAL-G22 [6]. The uncertainty due to the drift of the reference standard is denoted as $U_{\text {drift }}$ and the uncertainty due temperature difference is denoted as $U_{\text {temp }}$.

Table 1. Maximum relative expanded uncertainty of the reference force transducers $\left(u_{F s t d}\right)$ [4].

\begin{tabular}{|l|l|}
\hline Force transducer class & $U_{\text {Fstd }}(\%)$ \\
\hline 00 & 0.06 \\
\hline 0.5 & 0.12 \\
\hline 1 & 0.24 \\
\hline 2 & 0.45 \\
\hline
\end{tabular}

To evaluate the contribution of $U_{F s t d}$ on the uncertainty of reference force transducer, the value of $u_{F s t d}$ can be chosen either according to the class of the force transducer used (Table 1) or directly from the calibration certificate of the force transducer. The value of relative standard uncertainty of the force transducer $\left(u_{F s t d}\right)$ is estimated from the following equation (assuming normal probability distribution):

$$
U_{F s t d}= \pm \frac{u_{F s t d}}{2}
$$

The uncertainty due to the drift of the reference force transducer used in calibration can be estimated either by the manufacturer's specifications of the force transducer or by the long-term observations of the its calibration results [7]. The relative standard uncertainty due to the drift effect can be estimated according to the following equation (assuming rectangular probability distribution):

$$
U_{\text {drift }}= \pm \frac{u_{\text {drift }}}{\sqrt{3}}
$$

where $u_{d r i f t}$ is the relative drift uncertainty of the force transducer used (equals one half of the range of the evaluated drift value during the period from last calibration).

The calibrations of Charpy impact testing machines are usually performed in standard force metrology laboratories where the temperature is maintained within $20-25 \pm 2{ }^{\circ} \mathrm{C}$. The variation of the force transducer response from this small range of temperature variation (assuming rectangular distribution) shall be considered as follows:

$$
U_{t e m p}= \pm \frac{k_{t e m p} \Delta t}{\sqrt{3}}
$$

where $k_{\text {temp }}$ is the temperature coefficient of sensitivity per $1{ }^{\circ} \mathrm{C}$ of the calibrated force transducer (specified in the manufacturer's manual) and $\Delta t$ is half the expected temperature variations range during the calibration [7].

\subsubsection{Uncertainty due to reference Length measuring device, $U_{L}$}

The following equation describes the uncertainty of the reference length measuring device:

$$
U_{L}=\sqrt{U_{\text {Lstd }}^{2}+U_{\text {drift }}^{2}+U_{\text {temp }}^{2}}
$$

It is clear that for every instrument, at least 3 components should be used, uncertainty of the standard, uncertainty of the drift, and uncertainty of the temperature variation. To evaluate the contribution of $U_{L s t d}$ on the uncertainty of the Length measuring device, the value of $U_{\text {Lstd }}$ can be chosen directly from the calibration certificate of the force transducer. The value of relative standard uncertainty of the force transducer $\left(u_{L s t d}\right)$ is estimated from the following equation (assuming normal probability distribution):

$$
U_{\text {Lstd }}= \pm \frac{u_{\text {Lstd }}}{2}
$$

The uncertainty due to the drift of the Length measuring device used in calibration can be estimated either by the manufacturer's specifications of the Length measuring device or by the long-term observations of its calibration results. The relative standard uncertainty due to the drift effect can be estimated according to the following equation 
(assuming rectangular probability distribution):

$$
U_{\text {drift }}= \pm \frac{u_{\text {drift }}}{\sqrt{3}}
$$

where $u_{\text {drift }}$ is the relative drift uncertainty of the Length measuring device used (equals one half of the range of the evaluated drift value during the period from last calibration).

The calibrations of Charpy impact testing machines are usually performed in standard force metrology laboratories where the temperature is maintained within $20-25 \pm 2^{\circ} \mathrm{C}$. The variation of the Length measuring device from this small range of temperature variation (assuming rectangular distribution) shall be considered as follows [4]:

$$
U_{\text {temp }}= \pm \frac{k_{\text {temp }} \Delta t}{\sqrt{3}}
$$

\subsubsection{Uncertainty due reference Angle measuring device, $U_{a}$}

$U_{a}$ can be estimated as the same sequence of clause 2.1.2 as a dimensional metrology and its equation is:

$$
U_{\alpha}=\sqrt{U_{\alpha s t d}^{2}+U_{d r i f t}^{2}+U_{\text {temp }}^{2}}
$$

\subsection{Uncertainty due to Resolution effect, $U_{\text {res }}$}

The limit to the ability of an instrument to respond to small changes in the quantity being measured, can be considered as the uncertainty measurement due to resolution. In the digital instruments it can be considered as $\pm 1 / 2$ the scale value of the display. In an analog instrument it is determined by the practical ability to read the position of a scale. The value of the standard uncertainty due to resolution $\left(U_{r e s}\right)$ can be estimated from the following equation [8] (assuming rectangular distribution):

$$
U_{\text {res }}= \pm \frac{u_{\text {res }}}{\sqrt{3}}
$$

where $u_{\text {res }}$ is the uncertainty due to the resolution effect.

\subsection{Uncertainty due Indicated energy error, $U_{\text {Ind }}$}

For a machine has a nominal capacity $A_{N}$ Joules, verify the indicator graduations corresponding to $10,20,30,50$ or $60-80 \%$ of the initial nominal potential energy $A_{N}$ [2], then calculate the absorbed energy $A_{v}$ for each one. This followed by calculating the indicated energy error percentage $u_{\text {ind }}$. To do this, lift the pendulum driving the indicator in the rise direction until the indicator is on the graduation to be verified. Measure the angle $\beta$ of rise to within \pm $0.065^{\circ}$ as shown in Fig. 1. The energy absorbed is equal to:

$$
A_{V}=F \cdot L_{2}(\cos \beta-\cos \alpha)
$$

The difference between the energy indicator $A_{S}$ and the absorbed energy $A_{V}$ calculated on the basis of the measured values, shall not exceed $\pm 1 \%$ of the absorbed energy $A_{V}$ or $\pm 0.5 \%$ of the potential energy $A_{P}$. In each case, the greater value is permitted [2].

$$
u_{\text {ind }}=\left|\frac{A_{S}-A_{V}}{A_{V}}\right| \times 100 \leq 1.0
$$

(from 80 to $50 \%$ of $A_{P}$ )

$$
u_{\text {ind }}=\left|\frac{A_{S}-A_{V}}{A_{V}}\right| \times 100 \leq 0.5
$$

(below $50 \%$ of $A_{P}$ )

In case that the indicated energy error is within the specified error, the value as taken as the permissible one. If the indicated energy error exceeds the permissible values, it's recommended to make maintenance for the machine.

The value of the standard uncertainty due to indicated energy error $\left(U_{i n d}\right)$ can be estimated from the following equation (assuming triangular distribution):

$$
U_{\text {ind }}= \pm \frac{u_{\text {ind }}}{\sqrt{6}}
$$

\subsection{Uncertainty due to Drag of the pointer, $U_{\text {drag }}$}

Calculate the friction losses due to the drag of the pointer $p$. then, estimate the percentage of the losses $u_{d r a g}$. To 
do this, move the pointer to a position corresponding to a rise angle of zero, let the pendulum fall normally (fall angle a) but without the test piece in position and read off the rise angle $\beta_{1}$, or the energy $E_{1}$ directly.

Then, without resetting the pointer, let the pendulum fall a second time from the position corresponding to the fall angle and read off the new rise angle $\beta_{2}$, or the energy $E_{2}$ directly. When the scale is graduated in degrees, the friction losses of the pointer are equal to:

$$
P=F \cdot L_{2}\left(\cos \beta_{1}-\cos \beta_{2}\right)
$$

And when the scale is graduated in energy units, the friction losses of the pointer are equal to:

$$
P=E_{1}-E_{2}
$$

In this calculation, use the mean values of $\beta_{1}$ and $\beta_{2}$ (or $E_{1}-E_{2}$ ) from four determinations at least [2].

$$
u_{\text {drag }}=\frac{p}{A_{N}} \cdot 100
$$

The value of the standard uncertainty due to drag of the pointer $\left(U_{\text {drag }}\right)$ can be estimated from the following equation [8] (assuming rectangular distribution):

$$
U_{\text {drag }}=\frac{u_{\text {drag }}}{\sqrt{3}}
$$

\subsection{Uncertainty due to Bearing friction, $U_{\text {bear }}$}

After determining $\beta_{2}$ or the energy $E_{2}$ (see 2.4.), return the Pendulum to its initial position. Then, without readjusting the pointer, release the pendulum to allow 10 half-swings. After the pendulum has started its $11^{\text {th }}$ halfswing, move the pointer about $5 \%$ from its maximum reach and note the value of $\beta_{3}$. Friction losses, if the scale is graduated in degrees, in the bearings and as a result of air-resistance for a half-swing are:

$$
\dot{P}=\frac{1}{10} F \cdot L_{2}\left(\beta_{3}-\beta_{2}\right)
$$

Or friction losses, if the scale is graduated in energy, is equal to:

$$
\dot{P}=\frac{1}{10} F . L_{2}\left(E_{3}-E_{2}\right)
$$

Calculate the friction losses due to the bearing and as a result of air resistance $P$. then, estimate the percentage of the losses $u_{\text {bear. }}$.

$$
u_{\text {bear }}=\frac{\dot{P}}{A_{N}} \cdot 100
$$

The value of the standard uncertainty due to drag of the bearing friction $\left(U_{\text {bear }}\right)$ can be estimated from the following equation [9] (assuming rectangular distribution):

$$
U_{\text {bear }}=\frac{u_{\text {bear }}}{\sqrt{3}}
$$

According to [2], the total losses $P \dot{P}+P$ measured in this way shall not exceed $0.5 \%$ of the rated energy $A_{N}$, if the losses exceed that tolerance, the machine may need maintenance.

\subsection{Combined uncertainty of the machine, $U_{\text {comb }}$}

The value of the standard combined uncertainty of the machine, $U_{c o m b}$, can be estimated from the following equation:

$$
U_{\text {comb }}=\sqrt{U_{\text {ref }}^{2}+U_{\text {res }}^{2}+U_{\text {ind }}^{2}+U_{\text {drag }}^{2}+U_{\text {bear }}^{2}}
$$

\subsection{Expanded uncertainty of the machine, $U_{\text {exp }}$}

The value of the standard expanded uncertainty of the machine $\left(U_{\exp }\right)$ can be estimated from the following equation:

$$
U_{\text {exp }}=k \cdot U_{c o m b}
$$

Where $\mathrm{k}$ is a coverage factor 


\section{NUMERICAL EXAMPLE}

In order to gain familiarity with the principles set out in this study it may be useful to express all the previously mentioned affecting factors of uncertainty (Section 2) with the calibration results, the values of the individual standard uncertainties are calculated from the equations presented in Section 2.

For a machine the following data:

$A_{N}=300$ Joule, resolution=2 Joule $M=160.169 \mathrm{~N} . \mathrm{m}$, fall angle $\mathrm{a}=151.15^{\circ}, \mathrm{A}_{\mathrm{P}}=300.4596$ Joule, $\mathrm{E}_{1}, \mathrm{E}_{2}, \mathrm{E}_{3}=0.1,0.8$ Joule respectively, rise angles $\beta 132.5,119.4,108.152,86.31,51.32^{\circ}$ at 10 , at $10,20,30,50,80 \%$ of the $A_{N}$ capacity, respectively.

- Uncertainty of the reference standard force transducer used in the calibration (given in its calibration certificate): $U_{\text {Fstd }}=0.01 \%$ (for the whole range).

- Uncertainty due to long-term instability (drift) of the reference force transducer (evaluated from the longterm observations of the calibration results of the force transducer): $U_{\text {drift }}=0.0008 \%$ (for the whole range).

- Uncertainty due to temperature variation during calibrating the impact tester (given in the technical specifications of the reference force transducer used): $U_{\text {temp }}$ for $0.5^{\circ} \mathrm{C}$ temperature variation is found to be less than $0.0031 \%$ (for the whole range).

- Uncertainties of $U_{\text {Lstd, }}, U_{\text {drift, }}$ and $U_{\text {temp }}$ of the length measuring device are $0.015,0.0014$, and $0.01 \%$ respectively (for the whole range).

- Uncertainties of $U_{a s t d}, U_{d r i f t}$, and $U_{\text {temp }}$ of the angle measuring device are $0.001,0.0$, and $0.01 \%$ respectively (for the whole range).

- Uncertainty of used measuring device $U_{\text {ref }}$ is $0.149 \%$ (for the whole range).

- Uncertainty due to the resolution effect of the impact tester, when considering $u_{\text {res }}$ equals one fifth of the scale value: $U_{\text {res }}=0.71986,0.374523,0.25549,0.153348$, and $0.096068 \%$ at $10,20,30,50,80 \%$ of the $A_{N}$ capacity, respectively.

- Uncertainty due to indicated energy error the impact tester: $U_{\text {ind }}=0.28279,0.225875,0.053146,0.162208$, and $0.066451 \%$ at $10,20,30,50,80 \%$ of the $A_{N}$ capacity, respectively.

- Uncertainty due to drag of the pointer $U_{\text {drag }}=0.0192 \%$ (for the whole range).

- Uncertainty due to bearing friction is $U_{\text {bear }}=0.1539 \%$ (for the whole range).

- The relative combined standard uncertainty $\left(U_{\text {comb }}\right)$ can be estimated from equation $(28)=0.802781$, $0.487418,0.338213,0.310025$, and $0.244813 \%$ at $10,20,30,50,80 \%$ of the $A_{N}$ capacity, respectively.

- The relative expanded uncertainties $\left(U_{\text {exp }}\right)$ values for the calibrated impact tester are calculated from equation (29).

- The values of $U_{\text {exp }}$ are found to be 1.605562, 0.974836, 0.676426, 0.620051, and $0.489627 \%$ at $10,20,30$, $50,80 \%$ of the $A_{N}$ capacity, respectively, with a level of confidence of $95 \%$ ( $k=2$, assuming a normal distribution).

\section{CONCLUSIONS}

This study dealt with the main sources of uncertainty in the direct verification of the Charpy impact testers. These sources are: the uncertainty of reference force and length measuring devices and its long-term instability (drift), machine resolution, rated energy error, indicated energy error, losses due to the drag of the pointer, friction losses in the bearing and air resistance.

The study gives a numerical example as a guide to show how to estimate the expanded relative uncertainty in the calibration process. The numerical example has achieved its purpose and draw a guideline for the user to estimate the uncertainty of Charpy impact testers.

It's recommended, in the future work, to establish a similar proposal taking into consideration both indirect verification and instrumented machines.

\section{REFERENCES}

[1] Siewert, T. A., Manahan, M. R, McCowan, C. N., Holt, J. M., Marsh, E J., and Ruth, E. A., "The History and Importance of Impact Testing, Pendulum Impact Testing: A Century of Progress”, STP 1380, T. A. Siewert and M. E Manahan, Sr., Eds., American Society for Testing and Materials, West Conshohocken, PA, 2000.

[2] BSI DIN EN 10045, Part2, Verification of pendulum impact testing machines, direct verification method.

[3] BS EN ISO 148-2:2016, Metallic materials - Charpy pendulum impact test - Part 2: Verification of testing machines.

[4] A. A. Hawam, E. H. Hasan, G. Mohamed, M. Ahmadein, "The resolution uncertainty associated with digital indications revisited: The inclusion of the quantization effect and the impact of noise presence in the estimation process”, (2018) Metrologia, 55 (6), pp. 883-892.

[5] Kyle Wayne Strabala, "The Effects of Combined Compression and Aging on the Properties of Glassy Polycarbonate", Engineering Mechanics, Department of Engineering Mechanics Dissertations \& Theses, University of Nebraska, 
2009.

[6] EAL-G22-1996, Uncertainty of calibration results in force measurements.

[7] A. Sawla, Uncertainty of calibration results of tension/compression testing machines, PTB, March 1998.

[8] ISO GUM, Guide to the expression of uncertainty in measurement, 1993.

[9] Diedert Peschel and Dietmar Mauersberger, Determination of the friction of aerostatic radial bearings for the levermass system of torque standard machines, XIII IMEKO WOLD CONGRESS, September, 5-9, 1994, Torino, Italy. 